\title{
The descriptive epidemiology of total physical activity, muscle-strengthening exercises and sedentary behaviour among Australian adults - results from the National Nutrition and Physical Activity Survey
}

Jason A. Bennie ${ }^{1 *}$, Zeljko Pedisic ${ }^{1}$, Jannique G. Z. van Uffelen ${ }^{1}$, Joanne Gale ${ }^{2,3}$, Lauren K. Banting ${ }^{1}$, Ineke Vergeer ${ }^{1}$, Emmanuel Stamatakis ${ }^{3,4}$, Adrian E. Bauman ${ }^{2,3}$ and Stuart J. H. Biddle ${ }^{1}$

\begin{abstract}
Background: The current Australia's Physical Activity and Sedentary Behaviour Guidelines recommend that adults engage in regular moderate-to-vigorous-intensity physical activity (MVPA) and strength training (ST), and minimise time spent in sedentary behaviours (SB). However, evidence about the specific individual and concurrent distribution of these behaviours in Australia is scarce. Therefore, the aim of this study was to determine the prevalence and sociodemographic correlates of MVPA, ST and SB in a national-representative sample of Australian adults.

Methods: Data were collected using face-to-face interviews, as part of the National Nutrition and Physical Activity Survey 2011-12. The population-weighted proportions meeting the MVPA ( $\geq 150 \mathrm{~min} /$ week), ST ( $\geq 2$ sessions/week) and combined MVPA-ST guidelines, and proportions classified as having 'low levels of SB' ( $<480 \mathrm{~min} /$ day) were calculated, and their associations with selected sociodemographic and health-related variables were assessed using multiple logistic regression analyses. This was also done for those at potentially 'high-risk', defined as insufficient MVPA-ST and 'high-sedentary' behaviour.
\end{abstract}

Results: Out of 9345 participants (response rate $=77.0 \%$ ), aged 18-85 years, $52.6 \%$ (95\% Cl: $51.2 \%-54.0 \%$ ), $18.6 \%$ (95\% Cl: $17.5 \%-19.7 \%$ ) and $15.0 \%$ (95\% Cl: $13.9 \%-16.1 \%$ ) met the MVPA, ST and combined MVPA-ST guidelines, respectively. Female gender, older age, low/medium education, poorer self-rated health, being classified as underweight or obese, and being a current smoker were independently associated with lower odds of meeting the MVPA, ST and combined MVPA-ST guidelines. A total of $78.9 \%$ (95 \% Cl: $77.9 \%-80.0 \%$ ) were classified as having low levels of SB. Females, older adults and those with lower education were more likely to report lower levels of SB, whilst those with poor self-rated health and obese individuals were less likely to report lower levels of SB (i.e. $\mathrm{SB}=\geq 480 \mathrm{~min} / \mathrm{day}$ ). A total of $8.9 \%$ (95 \% Cl: 8.1\%-9.6\%) were categorised as individuals at potentially 'high-risk'. Those with poorer self-rated health, obese individuals, those aged $25-44$, and current smokers were more likely to be in the 'high risk' group.

(Continued on next page)

\footnotetext{
* Correspondence: jason.bennie@vu.edu.au

'Active Living and Public Health Program, Institute of Sport, Exercise and

Active Living (ISEAL), Victoria University, Melbourne, VIC, Australia

Full list of author information is available at the end of the article
} 
(Continued from previous page)

Conclusions: The large majority of Australian adults do not meet the full physical activity guidelines and/or report excessive SB. Our results call for public health interventions to reduce physical inactivity and SB in Australia, particularly among the subgroups at the highest risk of these unhealthy behaviours.

Keywords: Public health surveillance, Strength training, Physical activity, Sitting

\section{Background}

Non-communicable diseases (NCDs) are responsible for $68 \%$ of all deaths worldwide [1]. Physical inactivity is among the leading preventable causes of NCDs [2]. It is estimated to cause $6-10 \%$ of deaths related to coronary heart disease, diabetes, and breast and colon cancer [3]. It is the fourth ranked mortality risk factor; after hypertension, tobacco use and high blood glucose [3, 4], and it causes $9 \%$ of premature mortality [3]. Estimates based on self-report data suggest that globally $40 \%-60 \%$ of adults are insufficiently active for health [5]. Consequently, reducing rates of physical inactivity has been described as a key $21^{\text {st }}$ century public health challenge $[2,6,7]$.

Until recently, physical activity recommendations were primarily based around the accumulation of moderateto-vigorous-intensity aerobic physical activity (MVPA) (e.g. walking or jogging) $[8,9]$. However, evidence from epidemiological studies and controlled trials suggests that regular participation in muscle-strengthening activities (e.g. weight or resistance training) may provide additional benefits for musculoskeletal (e.g. reducing the risk of sarcopenia, osteoporosis and osteoarthritis) [10], metabolic (e.g. reducing the risk of metabolic syndrome and type 2 diabetes) [11, 12] and mental health (e.g. reducing the symptoms of anxiety) [10]. Moreover, recent studies have shown that, when compared to one activity mode alone, there may be greater metabolic health benefits of combining MVPA and muscle-strengthening activities [12-15].

In addition, sedentary behaviour (excessive sitting as opposed to insufficient physical activity) has recently emerged as a potential independent risk factor for poor health [16]. Prospective studies have shown that adults who accrue high volumes of sedentary behaviour are at a higher risk of developing chronic diseases such as type 2 diabetes [17-19] and cardiovascular disease [20], as well as at a higher risk of all-cause and disease-specific mortality [21-25]. Importantly, the association between prolonged sitting and detrimental health outcomes remains significant even after adjustment for time spent in MVPA [16, 26-28], indicating that, for optimal health benefits, people should both be active and limit their time spent in sedentary behaviour.

For the prevention and management of chronic diseases, leading global and national health authorities, such as the World Health Organization (WHO), the U.S. Department of Health and Human Services and the Australian Government, Department of Health, recommend that adults should participate in: (i) at least $150 \mathrm{~min} /$ week of moderate (e.g. brisk walking) or $75 \mathrm{~min} /$ week of vigorousintensity physical activity (e.g. jogging), or an equivalent combination of both, and (ii) 2 or more days per week of muscle-strengthening activities involving major muscle groups [7, 29-31]. Additionally, authorities within Australia and the U.S. recommend that adults limit time spent in sedentary behaviours $[29,30]$. At present there is no consensus on the threshold relationship between sedentary behaviour and poor health [16]. Hence, current sedentary behaviour reduction guidelines typically state that adults should 'limit prolonged periods of time spent in sedentary behaviours', and 'frequently break up long periods of sitting' [29, 30, 32].

Previous population-based studies have mostly focused on assessing the prevalence and correlates of MVPA [33-35]. Only few studies examined the proportion of people meeting the recommendations for strength training in national-representative samples.

Estimations range from 21.9 to $31.7 \%$ within the U.S and UK [36-40] and from 9.4 to $15.5 \%$ in Australia [41-43]. Data about meeting the combined MVPAmuscle-strengthening guidelines are even scarcer. Previous studies have shown that 18.2 to $20.6 \%$ of U.S. adults meet both MVPA and strength training guidelines [36, 44, 45], but no such estimates exist for the Australian population. Furthermore, international studies have reported that adults from different countries sit on average between 135 and $360 \mathrm{~min} /$ day, whilst the prevalence rates of total sitting time above $9 \mathrm{~h}$ /day ranged between 2.6 and $34.9 \%[46,47]$. No previous international or national studies have concurrently assessed the prevalence and correlates of MVPA, muscle-strengthening activities and sedentary behaviours, and clusters of these behaviours in a representative sample. Such data are essential to inform comprehensive interventions aimed to reduce physical inactivity and prolonged sitting, and to identify populations at the highest risk.

The aim of this study was, therefore, to determine the prevalence of adherence to the public health MVPA and muscle-strengthening activity guidelines, and high level of sedentarism among a population-representative sample of Australian adults and to investigate their sociodemographic and health-related correlates. 


\section{Methods}

This study accessed the data from the Australian Bureau of Statistics (ABS), National Nutrition and Physical Activity Survey (NNPAS) 2011-12. The NNPAS is a sub-component of the larger ABS Australian Health Survey (AHS), 2011-13, which is a national survey designed to provide detailed information on the health and wellbeing of the Australian population. Data from the NNPAS 2011-12 are are publicly accessible. The NNPAS 2011-12 was conducted between May 2011 and June 2012, and used a stratified multistage area sampling of private dwellings to ensure recruitment of a representative sample of Australian adults.

Detailed information on the survey design, data collection, and response rates can be found elsewhere [48]. The Australian Bureau of Statistics Research Ethics Committee approved all study protocols and written informed consent from all participants was obtained [48]. Upon given consent, data were obtained by face-to-face interviews in respondents' homes by trained interviewers using Computer Assisted Personal Interview (CAPI) system.

The initial sample size $(14,363$ households) was reduced to 12,366 dwellings after sample loss in the field stage. Of the remaining dwellings, 9519 (77.0 \%) fully or adequately responded to the first interview [48]. A total of 12,153 participants (aged 2-85 years) fully or adequately completed the survey. For the present study, we used data from adults aged 18-85 years, leaving a final sample of 9435 respondents. Each of the respondents was given a selection probability weight, which is reflective of how many people in the population they represent. More information on weighting in the NNPAS can be found on the ABS website [48].

\section{Measures and data management}

The questionnaire used in the NNPAS 2011-12 can be found elsewhere [49]. For the present study, we used the data on physical activity, strength/toning activities, sedentary behaviour and selected sociodemographic and healthrelated variables.

\section{Physical activity}

Self-reported physical activity levels were assessed using the Active Australia Survey which has been previously validated among adults and older adults. The survey has been shown to have adequate reliability for classifying participants into insufficiently/sufficiently active groups (Cohen's kappa $=0.50-0.52$ ) (ref), and adequate validity when assessed against pedometer step counts (Spearman rho $=0.42-0.43$ ) [50] and against accelerometers (Spearman rho $=0.52)$ [51]. Respondents reported the number of occasions (frequency) and estimated time spent (duration) in walking and other moderate-intensity activity (e.g. gentle swimming, social tennis doubles, golf), and vigorous physical activity (e.g. jogging, fast cycling, circuit training, competitive tennis) over the past week. It should be noted that these questions also covered participation in any moderate- or vigorous-intensity muscle-strengthening activity. The data were scored using established methods described in the guide for implementation of the Active Australia Survey [52]. The reported durations for each activity were summed to estimate the total time spent in MVPA. Based on the 2014 Australia's Physical Activity \& Sedentary Behaviour Guidelines for Adults [29], participants were dichotomised as either: (i) 'meeting the MVPA guidelines' ( $\geq 150$ moderate-intensity minutes/week or $\geq 75$ vigorous-intensity minutes/week or an equivalent combination of both), or (ii) 'not meeting the MVPA guidelines'.

\section{Muscle-strengthening activities}

To assess participation in muscle-strengthening activities, respondents were asked, "Including any activities already mentioned, in the last week did you do any strength or toning activities?". If they answered positively, they were further asked: "How many times did you do any strength or toning activities in the last week?". It should be noted that these questions covered the muscle-strengthening activities that participants already mentioned in their responses to questions about MVPA, and potentially any other muscle-strengthening activities that are not of moderate or vigorous intensity. Similar questions have previously shown adequate reliability (Cohen's kappa = $0.85-0.92$ ) [53], and have been used in population studies in Australia [41] and the US [38, 45]. For strength and toning activities, data were missing for 1 participant. According to the Australian Physical Activity and Sedentary Behaviour Guidelines [29] and consistent with previous population studies [36, 38, 41, 43, 45], the sample was dichotomised as either; (i) 'meeting the strength training guidelines' ( $\geq 2$ sessions/week), or (ii) 'not meeting the strength training guidelines' ( $<2$ sessions/week).

\section{Meeting the combined MVPA-strength training guidelines} Consistent with previous studies [36, 45, 54], based on the data about the participation in MVPA and musclestrengthening activities, the sample was dichotomised as either (i) meeting the combined MVPA-strength training guidelines ( $\geq 150$ MVPA minutes/week and $\geq 2$ sessions/ week of strength or toning activities) or (ii) not meeting the MVPA-strength training guidelines $(<150$ MVPA minutes/week or $<2$ sessions/week of musclestrengthening activities).

\section{Sedentary behaviour}

Respondents reported their time spent in sedentary behaviour during the last week within the following contexts: (i) sitting at work (ii); sitting for transport (including waiting for transport); (iii) sitting or lying down to watch 
television or videos; (iv) sitting or lying down to play electronic games; (v) sitting or lying down to use a computer or the internet; (vi) sitting or lying down to use a phone (including text messages and talking); and (vii) sitting or lying down to do other social or leisure activities (such as at a barbecue, for meals, at a cinema, etc.). Similar questions have previously shown adequate reliability and validity in adults $[55,56]$. Times spent sitting in these contexts were summed to calculate the total time spent in sedentary behaviour during the last week, and then divided by seven to report on minutes/day. Consistent with previous population studies [46], the sitting data were truncated at $960 \mathrm{~min} /$ day $(16 \mathrm{~h})$. A total of 45 out of 9435 cases $(0.5 \%$ of the total sample) were truncated for reporting sitting $>960 \mathrm{~min} /$ day and 32 (0.3\% of the sample) were missing data on sedentary behaviour altogether.

At present there is no official agreement around the threshold at which sitting is considered detrimental for health. However, a recent meta-analysis showed that sitting for more than $8 \mathrm{~h}$ /day significantly increases the risk of all-cause mortality [57]. While further research is needed to validate this threshold, the potential for using $8 \mathrm{~h} /$ day as a measure of excessive engagement in sedentary behaviour was recently discussed by public health experts in the development of Australian sedentary behaviour guidelines $[58,59]$. Therefore, in the present study, we considered $\geq 8 \mathrm{~h} /$ day $(\geq 480 \mathrm{~min} /$ day $)$ as a proxy measure of high engagement in sedentary behaviours, and classified participants as (i) 'low-sedentary' (<480 $\mathrm{min} /$ day) or (ii) 'high-sedentary' ( $\geq 480 \mathrm{~min} /$ day).

\section{Participants at 'high-risk' - clustering of insufficient MVPA} and strength training and high levels of sedentary behaviour Respondents were classified in the 'high-risk' group if they: (i) did not meet the MVPA guidelines ( $<150 \mathrm{~min} /$ week), and (ii) did not meet the strength training guidelines ( $<2$ sessions/week), and (iii) were in the 'high-sedentary' category ( $\geq 480 \mathrm{~min} /$ day).

\section{Sociodemographic and lifestyle variables}

Sociodemographic (sex, age, level of education) and health related variables (self-rated health and smoking status) were assessed during the interview using standard questions. Body Mass Index (BMI) was calculated based on objectively measured height and weight using standard methods and categorised into: $<18.5 \mathrm{~kg} / \mathrm{m}^{2}$ (underweight); from $\geq 18.5 \mathrm{~kg} / \mathrm{m}^{2}$ to $<25 \mathrm{~kg} / \mathrm{m}^{2}$ (normal/ acceptable weight range); from $\geq 25 \mathrm{~kg} / \mathrm{m}^{2}$ to $<30 \mathrm{~kg} / \mathrm{m}^{2}$ (overweight); and $\geq 30 \mathrm{~kg} / \mathrm{m}^{2}$ (obese). More detailed description of the sociodemographic and health-related data collected in NNPAS 2011-12 can be found elsewhere [49].

\section{Statistical analysis}

Percentages and their $95 \%$ confidence intervals (95\% CI) were calculated for the following categories: (i) meeting the MVPA guidelines; (ii) meeting the strength training guidelines; (iii) meeting the combined MVPA-strength training guidelines; (iv) 'low-sedentary' (<480 $\mathrm{min} /$ day); and (v) 'high-risk' (not meeting the MVPA guidelines/not meeting the strength training guidelines/'high-sedentary'). Chi-squared tests (either based on testing an overall association or trend depending on the variable) were used to test the unadjusted differences between the prevalence rates by selected sociodemographic (sex, age, level of education) and health-related variables (self-rated health, BMI, smoking status).

A series of multiple logistic regression analyses was used to assess the associations between sociodemographic and health-related variables and: (i) meeting/not meeting MVPA guidelines; (ii) meeting/not meeting the strength training guidelines; (iii) meeting/not meeting the combined MVPA-strength training guidelines; (iv) being 'low/high sedentary'; and (v) being/not being in the 'high-risk' group (not meeting the MVPA guidelines/ not meeting the strength training guidelines/'high-sedentary'). Each model included the following explanatory variables: sex (reference group [ref] = "male"); age (ref = "18-24 years"); education level (ref = "high"); self-reported general health status (ref = "excellent"); BMI (ref = "normal weight"); and smoking status (ref="never smoked"). Adjusted odds ratios and their $95 \%$ CIs were reported. Statistical analyses were conducted using IBM SPSS 22.0 statistical software (SPSS Inc. an IBM Company, Chicago, IL). For all statistical tests, a p-value of $<0.05$ was used to indicate statistical significance. All estimates were weighted using population weights provided by the ABS. Strata were not identifiable in the data due to confidentiality concerns, so the effect of the sample design on the accuracy of the estimates was accounted for by using the delete-a-group Jackknife method to calculate the standard error of the estimates. Replicate weights were provided by the ABS. More detailed information can be found on the ABS website [48].

\section{Results}

Data were available for 9435 adults aged 18-85 years (response rate $=77.0 \%$ ). As shown in Table 1, when compared to current Australian population estimates [51], the proportions of the NNPAS 2011-12 sample was largely concordant across most sociodemographic variables. In the current study, a total of $54.1 \%$ were female, $45.9 \%$ were aged $18-44$ years, $25.7 \%$ had high education levels (degree or higher degree), $51.5 \%$ reported either 'excellent' or 'very good' self-rated health, $34.3 \%$ had normal BMI status and $48.5 \%$ reported never smoking (Table 1). 
Table 1 Prevalence of meeting moderate to vigorous-intensity physical activity (MVPA) guidelines ${ }^{a}$, strength training guidelines ${ }^{b}$, the combined MVPA-strength training guidelines ${ }^{c}$ and being 'low-sedentary' ${ }^{\prime \prime}$ - overall and by sociodemographic and health-related factors

\begin{tabular}{|c|c|c|c|c|c|c|}
\hline & Current study $^{e}$ & $\begin{array}{l}\text { Population-weighted } \\
\text { estimates }^{f}\end{array}$ & $\begin{array}{l}\text { Met MVPA guideline }{ }^{a} \\
(n=9285)\end{array}$ & $\begin{array}{l}\text { Met strength training } \\
\text { guideline } \mathrm{E}^{\mathrm{b}}(n=9434)\end{array}$ & $\begin{array}{l}\text { Met both MVPA and strength } \\
\text { guidelines }{ }^{c}(n=9284)\end{array}$ & $\begin{array}{l}\text { 'Low-sedentary'd (<480 min/day) } \\
(n=9435)\end{array}$ \\
\hline & $n$ & $\mathrm{~N}$ & $\%(95 \% C l)^{9}$ & $\%(95 \% \text { Cl) })^{9}$ & $\%(95 \% \mathrm{Cl})^{9}$ & $\%(95 \% \mathrm{Cl})^{9}$ \\
\hline Total & 9435 & $17,042,208$ & $52.6(51.2-54.0)$ & $18.6(17.5-19.7)$ & $15.0(13.9-16.1)$ & $78.9(77.9-80.0)$ \\
\hline Sex & $n(\%)^{g}$ & $n(\%)^{9}$ & & & & \\
\hline Male & 4329 (45.9) & $8,406,261(49.3)$ & $55.0(52.8-57.3)$ & $20.7(19.2-22.2)$ & $16.9(15.4-18.4)$ & $75.0(73.2-76.8)$ \\
\hline Female & $5106(54.1)$ & $8,635,947(50.7)$ & $50.2(48.2-52.2)$ & $16.6(14.9-18.1)$ & $13.1(11.6-14.7)$ & $82.8(81.5-84.0)$ \\
\hline$p$-value & & & $0.002^{*}$ & $<0.001^{*}$ & $<0.001^{*}$ & $<0.001^{*}$ \\
\hline \multicolumn{7}{|l|}{ Age } \\
\hline 18-24 years & 780 (8.3) & $2,233,305(13.1)$ & $62.2(57.2-67.1)$ & $29.2(25.2-33.2)$ & $25.3(21.3-29.3)$ & $80.9(77.2-84.6)$ \\
\hline 25-34 years & $1617(17.1)$ & $3,152,930(18.5)$ & $58.7(54.7-62.6)$ & $23.3(19.9-26.6)$ & $19.0(15.6-22.3)$ & 73.4 (70.7-76.1) \\
\hline 35-44 years & $1843(19.5)$ & $3,147,104(18.5)$ & $53.4(50.3-56.6)$ & $18.5(16.4-20.5)$ & $15.3(13.4-17.3)$ & 76.8 (74.7-78.8) \\
\hline $45-54$ years & $1660(17.6)$ & $3,023,041(17.7)$ & $52.9(49.3-56.4)$ & $17.7(14.9-20.5)$ & $14.3(11.8-16.8)$ & 76.7 (73.9-79.5) \\
\hline 55-64 years & $1432(15.2)$ & $2,565,640(15.1)$ & $48.7(44.8-52.6)$ & $14.2(12.1-16.4)$ & $11.1(8.8-13.4)$ & 78.7 (75.6-81.7) \\
\hline $65-74$ years & $1255(13.3)$ & $1,680,712(9.9)$ & $46.5(42.4-50.7)$ & $12.4(10.2-14.6)$ & $8.3(6.3-10.4)$ & $87.7(85.2-90.1)$ \\
\hline$\geq 75$ years & $848(9.0)$ & $1,239,476(7.3)$ & $33.3(29.4-37.1)$ & $7.6(5.4-9.7)$ & $4.1(2.3-5.8)$ & $89.2(86.4-92.0)$ \\
\hline$p$-value & & & $<0.001^{*}$ & $<0.001^{*}$ & $<0.001^{*}$ & $<0.001^{*}$ \\
\hline \multicolumn{7}{|l|}{ Education level } \\
\hline Low (<Year 12 or no non-school qualification) & $2579(27.8)$ & $4,252,855(25.3)$ & $40.2(37.6-42.8)$ & $11.5(9.9-13.1)$ & $8.5(6.9-10.2)$ & $84.0(82.2-85.9)$ \\
\hline Medium (Year 12, diploma or certificate) & $4327(46.5)$ & $8,293,573(49.4)$ & $52.0(50.0-54.0)$ & $19.6(17.8-21.3)$ & $15.4(13.8-17)$ & $80.4(78.6-82.1)$ \\
\hline High (Degree or higher degree) & $2385(25.7)$ & $4,246,623(25.3)$ & $66.1(64-68.1)$ & $23.6(21.2-26.0)$ & $20.5(18.2-22.8)$ & $71.0(68.8-73.3)$ \\
\hline$p$-value & & & $<0.001^{*}$ & $<0.001^{*}$ & $<0.001^{*}$ & $<0.001^{*}$ \\
\hline \multicolumn{7}{|l|}{ Self-rated health } \\
\hline Excellent & $1537(16.3)$ & $2,919,514(17.1)$ & $67.9(65.4-70.4)$ & $29.7(26.1-33.3)$ & $26.1(22.5-29.7)$ & $79.5(76.5-82.4)$ \\
\hline Very good & $3223(35.2)$ & $6,172,432(36.2)$ & $57.4(55.1-59.7)$ & $21.5(19.3-23.7)$ & $17.9(15.8-19.9)$ & $79.8(78.0-81.5)$ \\
\hline Good & $2965(31.4)$ & $5,316,252(31.2)$ & $47.9(45.2-50.6)$ & $14.1(12.4-15.8)$ & $10.4(8.7-12)$ & $78.8(76.6-81.1)$ \\
\hline Fair & $1163(12.3)$ & $1,942,921(11.4)$ & $35.1(30.3-39.9)$ & $8.7(6.4-11.1)$ & $5.4(3.6-7.1)$ & $77.2(73.9-80.5)$ \\
\hline Poor & $477(4.7)$ & $691,090(4.1)$ & $30.5(24.1-36.9)$ & $8.4(4.5-12.3)$ & $5.2(2.1-8.3)$ & $75.0(67.8-82.2)$ \\
\hline$p$-value & & & $<0.001^{*}$ & $<0.001^{*}$ & $<0.001^{*}$ & 0.120 \\
\hline
\end{tabular}


Table 1 Prevalence of meeting moderate to vigorous-intensity physical activity (MVPA) guidelines ${ }^{a}$, strength training guidelines ${ }^{b}$, the combined MVPA-strength training guidelines ${ }^{c}$ and being 'low-sedentary' - overall and by sociodemographic and health-related factors (Continued)

\begin{tabular}{|c|c|c|c|c|c|c|}
\hline \multicolumn{7}{|l|}{ Body Mass Index $\left(\mathrm{kg} / \mathrm{m}^{2}\right)$} \\
\hline Underweight (<18.5) & $121(1.5)$ & $280,115(1.9)$ & $54.4(42.5-66.3)$ & $12.6(5.30-20.0)$ & $8.6(2.0-15.1)$ & $84.6(76.6-92.5)$ \\
\hline Normal (18.5-25) & $2735(34.3)$ & $5,262,928(36.1)$ & $59.4(57.0-61.8)$ & $24.2(21.5-26.9)$ & $20.5(18.1-22.9)$ & $80.3(78.2-82.3)$ \\
\hline Overweight (25-30) & $2898(36.4)$ & $5,266,721(36.1)$ & $56.1(53.6-58.6)$ & $20.3(18.2-22.4)$ & $15.8(13.8-17.9)$ & $79.0(77.1-80.9)$ \\
\hline Obese $(\geq 30)$ & $2203(27.7)$ & $3,776,994(25.9)$ & $45.0(42.4-47.6)$ & $12.4(10.7-14)$ & $9.7(8.2-11.3)$ & $76.7(74.2-79.1)$ \\
\hline$p$-value & & & $<0.001^{*}$ & $<0.001^{*}$ & $<0.001^{*}$ & $0.020^{*}$ \\
\hline \multicolumn{7}{|l|}{ Smoking status } \\
\hline Never smoked & $4573(48.5)$ & $8,776,577(51.5)$ & $53.7(51.8-55.6)$ & $19.0(17.4-20.5)$ & $15.5(14.1-16.9)$ & $79.5(77.9-81.2)$ \\
\hline Ex-smoker & 3077 (32.6) & $5,263,643(30.9)$ & $55.1(53.1-57.0)$ & $21.0(19.0-23.0)$ & $16.9(15.1-18.8)$ & $78.8(76.8-80.9)$ \\
\hline Current smoker & $1785(18.9)$ & $3,001,988(17.6)$ & $45.0(41.8-48.3)$ & $13.4(11.3-15.6)$ & $10.1(8.2-12.0)$ & $77.4(74.8-80.0)$ \\
\hline$p$-value & & & $<0.001^{*}$ & $0.003^{*}$ & $0.004^{*}$ & 0.140 \\
\hline
\end{tabular}

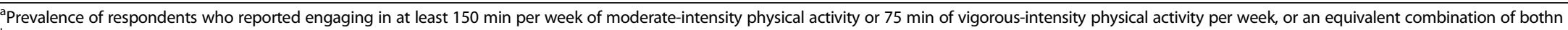

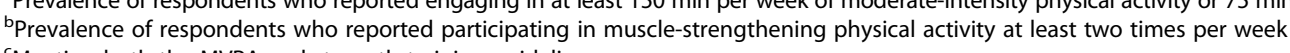

'Meeting both the MVPA and strength training guidelines

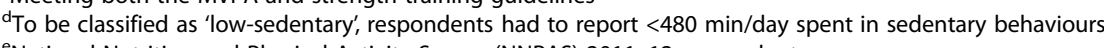

eNational Nutrition and Physical Activity Survey (NNPAS) 2011-12 respondents

'Replicate weights generated from the Australian Bureau of Statistics - Australian Health Survey: Users' Guide, 2011-13 - 4363.0.55.001

${ }^{9}$ Percentages are weighted and are given relative to the total number within each sociodemographic and health-related variable

${ }^{*} p<0.05: X^{2}$ test for overall association for sex and trend for the other sociodemographic and health-related variables 
Table 2 Adjusted odds ratios ${ }^{\mathrm{a}}(\mathrm{OR})$, and their $95 \%$ confidence intervals (95\% Cl), of being classified as meeting moderate to vigorous-intensity physical activity (MVPA) guidelines ${ }^{b}$, meeting the strength training guidelines ${ }^{c}$, meeting the combined MVPAstrength training guidelines ${ }^{d}$ and being classified as 'low-sedentary ${ }^{\prime e}$ - overall and by sociodemographic and health-related factors

\begin{tabular}{|c|c|c|c|c|}
\hline & $\begin{array}{l}\text { Met MVPA } \\
\text { guideline }^{b}\end{array}$ & $\begin{array}{l}\text { Met strength } \\
\text { training guideline }\end{array}$ & $\begin{array}{l}\text { Met both MVPA and } \\
\text { strength guidelines }\end{array}$ & $\begin{array}{l}\text { 'Low-sedentary' } \\
\text { (<480 min/day) }\end{array}$ \\
\hline Explanatory variable & OR $(95 \% \mathrm{Cl})$ & OR $(95 \% \mathrm{Cl})$ & OR $(95 \% \mathrm{Cl})$ & OR $(95 \% \mathrm{Cl})$ \\
\hline \multicolumn{5}{|l|}{ Gender (ref: male) } \\
\hline Female & $0.83(0.71-0.95)$ & $0.77(0.65-0.91)$ & $0.76(0.61-0.93)$ & $1.61(1.39-1.87)$ \\
\hline \multicolumn{5}{|l|}{ Age group (ref: 18-24 years) } \\
\hline $25-34$ years & $0.71(0.51-0.98)$ & $0.64(0.49-0.85)$ & $0.57(0.41-0.80)$ & $0.70(0.54-0.91)$ \\
\hline $35-44$ years & $0.57(0.43-0.75)$ & $0.46(0.36-0.60)$ & $0.44(0.32-0.60)$ & $0.85(0.63-1.14)$ \\
\hline $45-54$ years & $0.65(0.49-0.85)$ & $0.48(0.35-0.67)$ & $0.45(0.32-0.64)$ & $0.88(0.65-1.20)$ \\
\hline $55-64$ years & $0.55(0.41-0.75)$ & $0.37(0.29-0.49)$ & $0.33(0.23-0.45)$ & $1.03(0.78-1.36)$ \\
\hline $65-74$ years & $0.53(0.39-0.71)$ & $0.33(0.24-0.46)$ & $0.25(0.18-0.37)$ & $1.65(1.14-2.37)$ \\
\hline$\geq 75$ years & $0.36(0.25-0.52)$ & $0.21(0.14-0.32)$ & $0.14(0.08-0.24)$ & $2.25(1.37-3.70)$ \\
\hline \multicolumn{5}{|l|}{ Education level (ref: high [Degree or higher degree]) } \\
\hline Medium (Year 12, diploma or certificate) & $0.58(0.51-0.66)$ & $0.83(0.67-1.03)$ & $0.74(0.60-0.92)$ & $1.71(1.46-2.01)$ \\
\hline Low (<Year 12 and no non-school qualification) & $0.49(0.41-0.58)$ & $0.73(0.57-0.93)$ & $0.70(0.53-0.93)$ & $2.04(1.64-2.53)$ \\
\hline \multicolumn{5}{|l|}{ Self-rated health (ref: excellent) } \\
\hline Very good & $0.69(0.56-0.84)$ & $0.65(0.49-0.87)$ & $0.63(0.46-0.86)$ & $0.99(0.77-1.26)$ \\
\hline Good & $0.49(0.40-0.60)$ & $0.43(0.33-0.55)$ & $0.36(0.28-0.47)$ & $0.88(0.68-1.16)$ \\
\hline Fair & $0.35(0.26-0.46)$ & $0.32(0.23-0.46)$ & $0.24(0.16-0.36)$ & $0.78(0.58-1.05)$ \\
\hline Poor & $0.35(0.24-0.50)$ & $0.38(0.20-0.73)$ & $0.26(0.11-0.61)$ & $0.57(0.35-0.92)$ \\
\hline \multicolumn{5}{|l|}{ Body Mass Index (ref; normal [18.5-25]) } \\
\hline Underweight $(<18.5)$ & $0.81(0.50-1.33)$ & $0.39(0.21-0.73)$ & $0.31(0.14-0.68)$ & $1.33(0.69-2.58)$ \\
\hline Overweight (25-30) & $1.01(0.86-1.19)$ & $0.94(0.76-1.17)$ & $0.89(0.70-1.13)$ & $0.96(0.80-1.15)$ \\
\hline Obese $(\geq 30)$ & $0.76(0.64-0.89)$ & $0.64(0.50-0.81)$ & $0.65(0.50-0.85)$ & $0.77(0.63-0.93)$ \\
\hline \multicolumn{5}{|l|}{ Smoking status (ref; never smoked) } \\
\hline Ex-smoker & $1.29(1.14-1.47)$ & $1.39(1.16-1.68)$ & $1.47(1.20-1.80)$ & $0.94(0.76-1.15)$ \\
\hline Current smoker & $0.87(0.73-1.05)$ & $0.79(0.63-0.99)$ & $0.77(0.59-0.99)$ & $0.89(0.73-1.08)$ \\
\hline
\end{tabular}

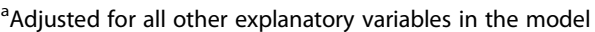

${ }^{\mathrm{b}}$ To meet the MVPA guideline respondents had to report engaging in at least 150 min per week of moderate-intensity physical activity or 75 min of vigorous-intensity physical activity per week, or an equivalent combination both

${ }^{C}$ To meet the strength training guideline, respondents had to report engaging in strength training at least two times per week

${ }^{\mathrm{d}}$ Meeting both the MVPA and strength training guidelines

'To be classified as 'low-sedentary', respondents had to report $<480 \mathrm{~min} /$ day spent in sedentary behaviours

For the total sample, the median time spent in MVPA was $150 \mathrm{~min} /$ week (95\% CI: 134-155, interquartile range [IQR]: 365 ). The majority of participants reported no participation in muscle-strengthening activities; hence the median number of sessions per week was 0 . Among the $22.7 \%$ of participants that did musclestrengthening activities, the mean number of sessions per week was 3.66 (95 \% CI: 3.34-23.86, SD = 3.01). The median time spent in sedentary behaviours was $308 \mathrm{~min} /$ day (95 \% CI: 305-314, IQR: 253).

As shown in Table 1, 52.6 \% (95 \% CI: $51.2 \%-54.0 \%$ ) of people met the MVPA guideline only, $18.6 \%$ (95\% CI: $17.5 \%-19.7 \%)$ met the strength training guideline (ST) only and $15.0 \%$ (95 \% CI: $13.9 \%-16.1 \%$ ) met both guidelines (MVPA-ST) (Table 1). Unadjusted analyses indicated significant differences between the proportions of participants meeting these guidelines across all sociodemographic and health-related variables $(p<0.001$ for almost all comparisons) (Table 1).

Accordingly, as shown in Table 2, in the adjusted analysis, all the selected sociodemographic and healthrelated variables showed significant overall associations with meeting/not meeting MVPA guideline, the strength training guideline, and the combination of both guidelines (overall $p<0.05$ for all explanatory variables). Specifically, females $(\mathrm{OR}=0.76,95 \% \mathrm{CI}$ : 0.61-0.93) were less likely to meet the combined MVPA-ST guidelines then males. When compared to adults aged 18-24 years, all other age groups were less likely to meet the combined MVPA-ST guidelines. The ORs ranged from 0.14 
(95 \% CI: $0.08-0.24$ ) for the oldest age group to 0.57 (95\% CI: 0.41-0.80) for those at the age of 25-34 years. Compared to adults reporting 'excellent' self-rated health, all other groups were less likely to meet the combined MVPA-ST guidelines. The ORs ranged from 0.26 (95\% CI: 0.11-0.61) for those reporting 'poor' health to 0.63 (95 \% CI: 0.46-0.86) for those reporting 'very good' health. When compared to those with high education, those with low $(\mathrm{OR}=0.70,95 \% \mathrm{CI}: 0.53-0.93)$ and medium education $(\mathrm{OR}=0.74,95 \% \mathrm{CI}$ : 0.60-0.92) were less likely to meet the combined MVPA-ST guidelines. Compared to those with normal BMI $\left(18.5-25 \mathrm{~kg} / \mathrm{m}^{2}\right)$, those who were classified as obese $(\mathrm{OR}=0.65,95 \% \mathrm{CI}$ : $0.50-0.85)$ and underweight $(\mathrm{OR}=0.31,95 \% \mathrm{CI}$ : 0.140.68 ) were less likely to meet the combined MVPA-ST guidelines. Interestingly, when compared to those who never smoked, ex-smokers were more likely ( $\mathrm{OR}=1.47$, 95 \% CI: 1.20-1.80), whilst current smokers were less likely $(\mathrm{OR}=0.77,95 \% \mathrm{CI}: 0.59-0.99)$ to meet the combined MVPA-ST guidelines.

A total of $78.9 \%$ (95\% CI: $77.9 \%-80.0 \%)$ were classified as 'low-sedentary' (Table 1). In the adjusted analysis, overall associations with sedentary behaviour categories were significant for all the explanatory variables (overall $p<0.001$ for all), except for smoking status (overall $p=0.49$ ). Specifically, when compared to the reference groups, females $(\mathrm{OR}=1.61,95 \% \mathrm{CI}: 1.39-1.87)$, adults aged 65-74 years $(\mathrm{OR}=1.65,95 \% \mathrm{CI}: 1.14-2.37)$ and $\geq 75$ years $(\mathrm{OR}=2.25$, $95 \%$ CI: $1.37-3.70)$ and those with the low $(\mathrm{OR}=2.04$, 95 \% CI: 1.64-2.53) or medium level of education (OR = 1.71, 95 \% CI: 1.46-2.01) were more likely to be classified as 'low-sedentary'. In contrast, when compared to the reference groups, obese individuals $(\mathrm{OR}=0.77,95 \% \mathrm{CI}$ : 0.63-0.93), those with poor self-related health $(\mathrm{OR}=0.57$, $95 \%$ CI: 0.35-0.92) and those aged 25-34 years (OR = 0.70, 95 \% CI: 0.54-0.91) were less likely to be classified as 'low-sedentary'.

Overall, $8.9 \%$ (95 \% CI: 8.1-9.6) of the sample were categorised as 'high-risk' with regards to their physical activity and sedentary behaviour (Table 3). In the adjusted analysis, compared to adults reporting 'excellent' health, those in other self-rated health categories were more likely to be in the 'high-risk' behaviour group (Table 4). The ORs ranged from 1.65 (95\% CI: 1.12-2.44) for 'very good' health category to 4.60 (95 \% CI: 2.897.33) for the 'poor' health category. When compared to the youngest age group, those aged 25-34 (OR $=1.91$, $95 \%$ CI: $1.27-2.87)$ and $35-44$ years $(\mathrm{OR}=2.09$, $95 \% \mathrm{CI}$ : $1.27-3.44)$ were more likely to be in the 'high-risk' group. Current smokers (OR $=1.37,95 \% \mathrm{CI}$ : 1.06-1.76) and obese individuals ( $\mathrm{OR}=1.44$, $95 \% \mathrm{CI}$ : 1.04-1.98) were more likely to be classified in the 'high-risk' group than those who never smoked and those with normal weight, respectively.

\section{Discussion}

This paper is the first to concurrently establish the prevalence and correlates of MVPA, strength training and sedentary behaviour among a national-representative sample of Australian adults.

The key finding of this study is that the vast majority (85\%) of Australian adults did not meet the full physical activity guidelines that incorporate both MVPA and strength training. Previous studies assessing the prevalence of physical inactivity among Australian adults have been solely based on MVPA levels, and show that $40 \%-$ $50 \%$ of Australians are insufficiently active for health [5, 35, 60]. However, our results suggest that when combining strength training and MVPA levels, the prevalence of physical inactivity far exceeds previous estimates. In fact, our findings suggest that estimating population adherence to the physical activity recommendations using only MVPA data may be largely misleading and may not reveal the true extent of the problem of inactivity.

When compared to the findings from U.S. populationbased studies [36, 44, 45], fewer Australian adults met both the MVPA and strength training guidelines (15.0\% vs. $18.2 \%-20.6 \%)$. Our prevalence estimate for meeting the strength training guidelines is slightly higher than in previous Australian studies. Among 5, 800 participants from the Australian Diabetes, Obesity and Lifestyle (AusDiab) study [42], 5, 700 from a sample of older Australian adults ( $\geq 65$ years) [45], and $\sim 1,200$ adults from regional Australia [41], the prevalence range was $9.4 \%-15.5 \%$. However, when compared to data from previous U.S. and UK studies, our findings suggest that strength training participation among Australian adults is somewhat lower (18.6\% vs. $21.9 \%-31.7 \%)$ [36-40]. Furthermore, when compared to the most recent physical activity prevalence data from the WHO Global Health Observatory [35], the proportions of Australians in our sample meeting the MVPA guidelines is somewhat similar.

The importance of physical inactivity from a clinical perspective was highlighted in a recent report released by the Australian Institute of Health and Welfare [61]. In that report, physical inactivity was identified as the most prevalent risk factor for cardiovascular disease (57 \% [note: based on insufficient MVPA levels only]), followed by high cholesterol $(32.8 \%)$ and high blood pressure (30\%). Given the substantial health benefits associated with regular participation in both MVPA and strength training, the low prevalence of Australian adults meeting these guidelines is of serious concern for public health. Comprehensive approaches are needed to promote and support both aspects of physical activity concurrently at the population level. 
Table 3 Percentage of Australian adults classified as 'high-risk' ${ }^{\text {'a }}$ based on reporting insufficient moderate to vigorous-intensity physical activity (MVPA), insufficient strength training participation, and high sedentary time - overall and by sociodemographic and health-related factors

\begin{tabular}{|c|c|c|c|}
\hline & $\begin{array}{l}\text { Current } \\
\text { study }\end{array}$ & $\begin{array}{l}\text { Population- } \\
\text { weighted } \\
\text { estimates }{ }^{c}\end{array}$ & 'High-risk'a \\
\hline & $n$ & $\mathrm{~N}$ & $\%(95 \% \mathrm{Cl})^{\mathrm{e}}$ \\
\hline Total & 867 & $1,500,637$ & $8.9(8.1-9.6)$ \\
\hline \multicolumn{4}{|l|}{ Sex } \\
\hline Male & 450 & 805,012 & $9.6(8.5-10.7)$ \\
\hline Female & 417 & 695,626 & $8.1(7.0-9.2)$ \\
\hline$p$-value & & & $0.04^{*}$ \\
\hline \multicolumn{4}{|l|}{ Age } \\
\hline $18-24$ years & 53 & 136,364 & $6.1(4.1-8.2)$ \\
\hline $25-34$ years & 146 & 287,331 & $9.1(7.6-10.7)$ \\
\hline $35-44$ years & 172 & 325,118 & $10.4(8.7-12.0)$ \\
\hline $45-54$ years & 165 & 271,123 & $9.0(7.2-10.9)$ \\
\hline $55-64$ years & 158 & 263,115 & $10.3(7.9-12.7)$ \\
\hline $65-74$ years & 108 & 119,006 & $7.1(5.3-9.0)$ \\
\hline$\geq 75$ years & 65 & 98,581 & $8.1(5.8-10.4)$ \\
\hline$p$-value & & & $0.49^{e}$ \\
\hline \multicolumn{4}{|l|}{ Education level } \\
\hline $\begin{array}{l}\text { Low (<Year } 12 \text { and no } \\
\text { non-school qualification) }\end{array}$ & 260 & 432,408 & $10.2(8.8-11.7)$ \\
\hline $\begin{array}{l}\text { Medium (Year 12, diploma } \\
\text { or certificate) }\end{array}$ & 388 & 726,351 & $8.8(7.5-10.1)$ \\
\hline High (Degree or higher degree) & 210 & 322,979 & $7.6(6.3-8.9)$ \\
\hline$p$-value & & & $0.01^{*}$ \\
\hline \multicolumn{4}{|l|}{ Self-assessed health } \\
\hline Excellent & 1532 & 291,2947 & $4.9(3.5-6.3)$ \\
\hline Very good & 3299 & 612,7479 & $7.4(6.3-8.5)$ \\
\hline Good & 2950 & $5,297,893$ & $9.4(7.9-11.0)$ \\
\hline Fair & 1155 & $1,927,885$ & $14.3(11.4-17.2)$ \\
\hline Poor & 443 & 684,920 & $18.8(13.8-23.8)$ \\
\hline$p$-value & & & $<0.001^{*}$ \\
\hline \multicolumn{4}{|l|}{ Body Mass Index $\left(\mathrm{kg} / \mathrm{m}^{2}\right)$} \\
\hline Underweight $(<18.5)$ & 121 & 280,115 & $7.2(1.0-13.4)$ \\
\hline Normal (18.5-25) & 2723 & $5,228,776$ & $7.1(5.8-8.5)$ \\
\hline Overweight (25-30) & 2889 & $5,253,598$ & $7.1(5.70-8.50)$ \\
\hline Obese $(\geq 30)$ & 2191 & $3,757,075$ & $12.3(10.3-14.3)$ \\
\hline$p$-value & & & $0.002^{*}$ \\
\hline
\end{tabular}

Smoking status

Never smoked
$373 \quad 701,268$

$8.0(7.0-9.1)$
Table 3 Percentage of Australian adults classified as 'high-risk', based on reporting insufficient moderate to vigorous-intensity physical activity (MVPA), insufficient strength training participation, and high sedentary time - overall and by sociodemographic and health-related factors (Continued)

\begin{tabular}{llll}
\hline Ex-smoker & 276 & 438,185 & $8.4(7.1-9.6)$ \\
Current smoker & 218 & 361,185 & $12.1(10.2-14)$ \\
$p$-value & & & $<0.001^{*}$
\end{tabular}

a'High risk' group defined as: insufficient MVPA ( $<150$ min/week); AND insufficient strength training participation ( $<2$ sessions/week); AND being classified as 'high-sedentary' ( $\geq 480 \mathrm{~min} /$ day)

${ }^{\mathrm{b}}$ National Nutrition and Physical Activity Survey (NNPAS) 2011-12 respondents ${ }^{\mathrm{C}}$ Replicate weights generated from the Australian Bureau of Statistics -

Australian Health Survey: Users' Guide, 2011-13 - 4363.0.55.001

dPercentages are weighted and are given relative to the total number within each sociodemographic and health-related variable

eWhereas the trend test was not significant for age group, the $X^{2}$ test for overall association was 0.020

${ }^{*} p<0.05: X^{2}$ test for overall association for sex and trend for the other sociodemographic and health-related variables

The sociodemographic correlates of strength training and MVPA observed in this study are consistent with existing data. Previous research has shown that older age, lower education levels and having poor health are associated with a lower prevalence of strength training [62], MVPA [33] and combined strength training and MVPA [37]. Our data underscore the importance of targeting these population groups in health promotion strategies. Particularly concerning was the finding that over $80 \%$ of Australians do not engage in sufficient strength training. Research suggests that, independent of MVPA, strength training has beneficial outcomes which are important for health and wellbeing, such as prevention and treatment of diabetes $[12,63]$ and cognitive decline [10] and improvements and maintenance of skeletal muscle mass/strength [10], bone mineral density [64] and physical functioning [65]. More research is now needed to examine the key factors influencing strength training participation [66]. However, when contrasted the decades of research examining the correlates and predictors of leisure-time physical activity [33, 67, 68], comparatively little is known about the key factors influencing strength training.

This study examined only a small number of potential correlates of MVPA and muscle-strengthening activities. Future studies should move beyond this and examine other potential socio-demographic, lifestyle, psychological (e.g. motivation, intentions), social (e.g. social support, perceived social norms) and environmental factors (e.g. access to facilities, affordability) influencing MVPA and strength training participation in the Australian adult population. Additionally, given that strength training often requires equipment and specific knowledge in exercise instruction, future research may be needed to evaluate the effectiveness of incentives, such as subsidising equipment (e.g. resistance bands, 
Table 4 Adjusted odds ratios ${ }^{a}(\mathrm{OR})$, and their $95 \%$ confidence intervals $(95 \% \mathrm{Cl}$ ), of being classified as 'high-risk', based on reporting insufficient moderate to vigorous-intensity physical activity (MVPA), insufficient strength training participation, and high sedentary time

\begin{tabular}{ll}
\hline Explanatory variable & OR (95 \% Cl) \\
\hline Gender (ref: male) & $0.84(0.70-1.01)$ \\
Female & \\
Age group (ref: 18-24 years) & $1.91(1.27-2.87)$ \\
25-34 years & $2.09(1.27-3.44)$ \\
35-44 years & $1.39(0.91-2.12)$ \\
45-54 years & $1.64(0.98-2.75)$ \\
$55-64$ years & $1.26(0.80-1.98)$ \\
65-74 years & $1.06(0.58-1.96)$ \\
$\geq 75$ years & \\
Education level (ref: high, completed year & \\
12 or equivalent) & $1.03(0.77-1.39)$ \\
Medium (completed year 10-11) & $1.07(0.79-1.46)$ \\
Low (completed year 9 or less) & \\
Self-assessed health (ref: excellent) & $1.65(1.12-2.44)$ \\
Very good & $2.10(1.39-3.16)$ \\
Good & $2.96(1.86-4.73)$ \\
Fair & $4.60(2.89-7.33)$ \\
Poor & \\
Body Mass Index (ref; normal [18.5-25]) & $0.99(0.39-2.51)$ \\
Underweight (<18.5) & $1.44(1.04-1.98)$ \\
Overweight (25-30) & \\
Obese ( $\geq 30)$ & $(1.06-1.76)$ \\
Ex-smoking status (ref; never smoked) & \\
Current smoker & \\
\hline
\end{tabular}

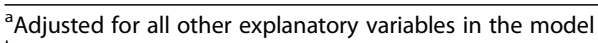

${ }^{b}{ }^{\prime} H i g h$ risk' group defined as: insufficient MVPA ( $<150 \mathrm{~min} /$ week); AND

insufficient strength training participation ( $<2$ sessions/week); AND being classified as 'high-sedentary' ( $\geq 480 \mathrm{~min} /$ day)

dumbbells) and fitness/health club memberships, to increase muscle-strengthening activity participation rates.

The percentage of participants classified as 'low-sedentary' ( $<480 \mathrm{~min} /$ day) was somewhat lower than in a large Australian study among $\sim 6700$ women [22] and in a study from Japan among $~ 83,000$ middle-aged and older adults [69] (78.9\% vs. $83.5 \%$ and $92.7 \%$, respectively). These slight variations are likely to be explained by differences in samples and the use of diverse sedentary behaviour assessment tools. . Being classified as 'high-sedentary' was associated with overweight/obesity, higher education and poorer self-rated health. These findings are somewhat similar with research on the correlates of high volumes of siting, which show a relationship between high sitting volumes and poor health status, high BMI and high education levels $[46,70]$. The inverse associations between age and sitting time are consistent with previous largescale studies using similar self-report measures [46, 47]. Furthermore, there are a number of other potential correlates of sedentary behaviour [71]; however their analysis was beyond the scope of this paper.

To our knowledge, this is the first study to determine the proportion of the population at potentially high risk, based on the clustering of 'not meeting the MVPA guidelines', 'not meeting the strength training guidelines' and being classified as 'high-sedentary'. Assuming that there may be cumulative health risks associated with insufficient physical activity and excessive sedentary behaviour, this population group are of a particular public health concern. The fact that there are up to $18.8 \%$ of people at the 'high-risk' in different population subgroups, should motivate public health stakeholders to put even greater efforts in targeting prevention. The odds of being classified as 'high-risk' were 4.6 times higher among adults who reported 'poor' health when compared to those who reported 'excellent' health. Those classified as obese had $\sim 44 \%$ higher odds to be in the 'high-risk' group than individuals with normal BMI. These findings are somewhat consistent with MVPA/strength training correlates research [33, 45], and further underscore the need to target such groups in health promotion strategies. Further studies using this clustering approach of combining those with insufficient MVPA/strength training levels and high levels of sedentary behaviour are needed to compare and contrast our findings.

Strengths of this study include the involvement of a large national-representative sample of Australian adults [48]. Furthermore, the current study enabled assessment of physical activity and sedentary behaviour across a variety of sociodemographic and health-related variables. A further strength was the use of standardised MVPA, strength training and sedentary behaviour assessment instruments, which allowed for comparisons with other studies.

Limitations of the study were the use of self-report measures of MVPA, strength training and sedentary behaviour, which may have resulted in recall bias [72]. To improve the validity of estimates, future studies might consider using accelerometers and inclinometers to assess time spent in MVPA and sedentary behaviours/ sitting, and time-use diaries to assess strength training participation. Nevertheless, for public health surveillance, standardised self-report instruments seems to be the method of choice for assessing the physical activity and sedentary behaviour levels [73]. Furthermore, no data was available on breaks in sitting time. Some of the participants classified as 'high sedentary' might have reduced health risks of prolonged sitting by taking regular 
breaks in sedentary behaviour [74]. The frequency of breaks in sitting time among Australian adults remains to be explored in future national surveys. A further limitation to this study was that, given its cross-sectional design, the direction of causality could not have been determined. For example, it may be that obesity lead to increased sitting time [75], but it is also possible that the direction of causality was opposite [76]. Furthermore, this study investigated only a small number of selected sociodemographic and lifestyle variables related to MVPA, muscle-strengthening activity and sedentary behaviour. Future studies are needed to identify and describe other potential correlates.

\section{Conclusions}

This study showed that the vast majority of Australian adults do not meet the full PA recommendation that incorporates both MVPA and strength training. In particular, our findings showing the low levels of strength training among Australian adults warrant attention. While strength training is an important component of physical activity-related health, it has practically been ignored by public health approaches to chronic disease prevention. In addition to the continual population monitoring of strength training and MVPA levels, public health interventions should target subgroups at the highest risk of low participation levels in these physical activity-related behaviours (e.g. adults aged 55+ years, females and those with low-education levels).

Furthermore, it seems that interventions to reduce sitting time should target males, younger age groups, those with high level of education, obese individuals and those with poor self-rated health. Finally, multifaceted interventions may be needed for those with poorer self-rated health, obese individuals, those aged 25-44, and current smokers, as they are at the highest risk of high sedentary behaviour combined with insufficient MVPA and musclestrengthening activity.

\section{Competing interests}

JAB declares he has a research fellowship part-funded by Fitness Australia, a not-for-profit, member-based industry association representing the interests of over 30,000 Australian registered exercise professionals, fitness service providers and industry suppliers. SJHB declares he has been a consultant to Fitness First. JAB and SJHB can confirm that these funders had no role in study design, data collection and analysis, decision to publish, or preparation of the manuscript. All other authors declare that they have no competing interests.

\section{Authors' contributions}

$J A B, Z P$ and $A E B$ conceptualised the study and developed the initial research plan. JG and JAB conducted the data analysis. JAB drafted the initial manuscript. ZP provided expertise on the analysis and interpretation of data and assisted in drafting the manuscript. SJHB, JVU, $L K B, I V, E S$ and $A E B$ provided guidance on the study and critically reviewed the manuscript. All authors read and approved the final version of the manuscript for publication.

\section{Acknowledgements}

This study was partially funded by the Victoria University, ISEAL Research Program Strategic Project Grant (project title: "Epidemiological assessment of muscle strengthening exercise participation in Australia"). JAB wishes to thank Fitness Australia for part-funding his research fellowship. We thank all National Nutrition and Physical Activity Survey participants for their generous contributions. We also thank staff at the Australian Bureau of Statistics,

Canberra, Melbourne and Sydney offices for their time and support during the Data Laboratory sessions.

\section{Author details}

${ }^{1}$ Active Living and Public Health Program, Institute of Sport, Exercise and Active Living (ISEAL), Victoria University, Melbourne, VIC, Australia.

${ }^{2}$ Prevention Research Collaboration, Sydney School of Public Health, University of Sydney, Sydney, NSW, Australia. ${ }^{3}$ Charles Perkins Centre, University of Sydney, Sydney, NSW, Australia. ${ }^{4}$ Exercise and Sport Sciences, University of Sydney, Sydney, NSW, Australia.

\section{Received: 4 August 2015 Accepted: 14 January 2016}

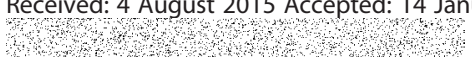

\section{References}

1. WHO. Global status report on noncommunicable diseases. Geneva, Switzerland: World Health Organization; 2014.

2. Blair SN. Physical inactivity: the biggest public health problem of the 21st century. Br J Sports Med. 2009;43(1):1-2.

3. Lee IM, Shiroma EJ, Lobelo F, Puska P, Blair SN, Katzmarzyk PT. Effect of physical inactivity on major non-communicable diseases worldwide: an analysis of burden of disease and life expectancy. Lancet. 2012;380(9838): 219-29.

4. WHO. Global health risks: mortality and burden of disease attributable to selected major risks. Geneva, Switzerland: World Health Organization; 2009.

5. Hallal PC, Andersen LB, Bull FC, Guthold R, Haskell W, Ekelund U. Global physical activity levels: surveillance progress, pitfalls, and prospects. Lancet. 2012;380(9838):247-57.

6. Booth FW, Gordon SE, Carlson CJ, Hamilton MT. Waging war on modern chronic diseases: primary prevention through exercise biology. J Appl Physiol (1985). 2000;88(2):774-87.

7. WHO. Global Recommendations on Physical Activity for Health. Geneva, Switzerland: World Health Organization; 2010.

8. Blair SN, LaMonte MJ, Nichaman MZ. The evolution of physical activity recommendations: how much is enough? The American journal of clinical nutrition. 2004;79(5):913s-20s.

9. Oja P, Titze S. Physical activity recommendations for public health: development and policy context. EPMA J. 2011;2(3):253-9.

10. Westcott WL. Resistance training is medicine: effects of strength training on health. Current sports medicine reports. 2012;11(4):209-16.

11. Churilla JR, Magyari PM, Ford ES, Fitzhugh EC, Johnson TM. Muscular strengthening activity patterns and metabolic health risk among US adults*. Journal of diabetes. 2012;4(1):77-84.

12. Grøntved A, Rimm EB, Willett WC, Andersen LB, Hu FB. A prospective study of weight training and risk of type 2 diabetes mellitus in men. Arch Intern Med. 2012;172(17):1306-12.

13. Dâmaso AR, da Silveira Campos RM, Caranti DA, de Piano A, Fisberg M, Foschini $D$, et al. Aerobic plus resistance training was more effective in improving the visceral adiposity, metabolic profile and inflammatory markers than aerobic training in obese adolescents. J Sports Sci. 2014;32(15): $1435-45$.

14. Mann S, Beedie C, Jimenez A. Differential effects of aerobic exercise, resistance training and combined exercise modalities on cholesterol and the lipid profile: review, synthesis and recommendations. Sports Med. 2014; 44(2):211-21.

15. Sousa N, Mendes R, Abrantes C, Sampaio J, Oliveira J. Long term effects of aerobic training versus combined aerobic and resistance training in modifying cardiovascular disease risk factors in healthy elderly men. Geriatrics \& gerontology international. 2013;13(4):928-35.

16. Bauman, Chau J, Ding D, Bennie J. Too much sitting and cardio-metabolic risk: an update of epidemiological evidence. Current Cardiovascular Risk Reports. 2013;7(4):293-8. 
17. Dunstan DW, Salmon J, Owen N, Armstrong T, Zimmet PZ, Welborn TA, et al. Associations of TV viewing and physical activity with the metabolic syndrome in Australian adults. Diabetologia. 2005;48(11):2254-61.

18. Ford ES, Schulze MB, Kroger J, Pischon T, Bergmann MM, Boeing $H$. Television watching and incident diabetes: findings from the European prospective investigation into cancer and nutrition-Potsdam study. Journal of diabetes. 2010;2(1):23-7.

19. Hu FB, Leitzmann MF, Stampfer MJ, Colditz GA, Willett WC, Rimm EB. Physical activity and television watching in relation to risk for type 2 diabetes mellitus in men. Arch Intern Med. 2001;161(12):1542-8.

20. Ford ES, Caspersen CJ. Sedentary behaviour and cardiovascular disease: a review of prospective studies. Int J Epidemiol. 2012;41(5):1338-53.

21. Koster A, Caserotti P, Patel KV, Matthews CE, Berrigan D, Van Domelen DR, et al. Association of sedentary time with mortality independent of moderate to vigorous physical activity. PLoS One. 2012;7(6):e37696.

22. Pavey TG, Peeters GG, Brown WJ. Sitting-time and 9-year all-cause mortality in older women. Br J Sports Med. 2015;49(2):95-9.

23. Stamatakis E. Association between sedentary behaviour and cardiometabolic risk factors in older adults. J Aging Phys Act. 2012;20:S301-1.

24. van der Ploeg HP, Chey T, Korda RJ, Banks E, Bauman A. Sitting time and all-cause mortality risk in 222497 Australian adults. Arch Intern Med. 2012; 172(6):494-500.

25. Wijndaele K, Brage S, Besson H, Khaw KT, Sharp SJ, Luben R, et al. Television viewing time independently predicts all-cause and cardiovascular mortality: the EPIC Norfolk study. Int J Epidemiol. 2011;40(1):150-9.

26. Biswas A, Oh Pl, Faulkner GE, Bajaj RR, Silver MA, Mitchell MS, et al. Sedentary time and its association with risk for disease incidence, mortality, and hospitalization in adults: a systematic review and meta-analysis. Ann Intern Med. 2015;162(2):123-32.

27. Thorp AA, Owen N, Neuhaus M, Dunstan DW. Sedentary behaviors and subsequent health outcomes in adults a systematic review of longitudinal studies, 1996-2011. Am J Prev Med. 2011:41(2):207-15.

28. Wilmot EG, Edwardson CL, Achana FA, Davies MJ, Gorely T, Gray LJ, et al. Sedentary time in adults and the association with diabetes, cardiovascular disease and death: systematic review and meta-analysis. Diabetologia 2012.

29. Australia's physical activity and sedentary behaviour guidelines (adults) [http://www.health.gov.au/internet/main/publishing.nsf/Content/pasb].

30. Garber CE, Blissmer B, Deschenes MR, Franklin BA, Lamonte MJ, Lee IM, et al. American College of Sports Medicine position stand. Quantity and quality of exercise for developing and maintaining cardiorespiratory, musculoskeletal, and neuromotor fitness in apparently healthy adults: guidance for prescribing exercise. Med Sci Sports Exerc. 2011:43(7):1334-59.

31. U.S Department of Health and Human Services: 2008 Physical activity guidelines for Americans. In. Washington, USA; 2008.

32. UK Department of Health. In: Health Do, editor. UK physical activity guidelines. London, UK: UK Goverment; 2011.

33. Bauman AE, Reis RS, Sallis JF, Wells JC, Loos RJ, Martin BW. Correlates of physical activity: why are some people physically active and others not? Lancet. 2012;380(9838):258-71.

34. Dumith SC, Hallal PC, Reis RS, Kohl 3rd HW. Worldwide prevalence of physical inactivity and its association with human development index in 76 countries. Prev Med. 2011:53(1-2):24-8.

35. Global health observatory data repository [http://apps.who.int/ghodata/].

36. Carlson SA, Fulton JE, Schoenborn CA, Loustalot F. Trend and prevalence estimates based on the 2008 Physical activity guidelines for Americans. Am J Prev Med. 2010;39(4):305-13.

37. Centers for Disease Control and Prevention. Adult participation in aerobic and muscle-strengthening physical activities-United States. MMWR Morbidity and mortality weekly report 2013. 2011;62(17):326-30.

38. Loustalot F, Carlson SA, Kruger J, Buchner DM, Fulton JE. Musclestrengthening activities and participation among adults in the United States. Res Q Exerc Sport. 2013;84(1):30-8

39. Schoenborn CA, Stommel M. Adherence to the 2008 adult physical activity guidelines and mortality risk. Am J Prev Med. 2011;40(5):514-21.

40. UK Health and Social Care Information Centre. In: Centre $\mathrm{HaSCl}$, editor. Health Survey for England 2012: Summary of key findings. UK: Leeds; 2012

41. Humphries B, Duncan MJ, Mummery WK. Prevalence and correlates of resistance training in a regional Australian population. Br J Sports Med. 2010;44(9):653-6.
42. Merom D, Pye V, Macniven $R$, van der Ploeg $H$, Milat A, Sherrington $C$, et al. Prevalence and correlates of participation in fall prevention exercise/ physical activity by older adults. Prev Med. 2012;55(6):613-7.

43. Minges KE, Magliano DJ, Owen N, Daly RM, Salmon J, Shaw JE, et al. Associations of strength training with impaired glucose metabolism: the AusDiab Study. Med Sci Sports Exerc. 2013;45(2):299-303.

44. Schoenborn C, Adams PF, Peregoy JA. Health behaviors of adults: United States, 2008-2010. In. Edited by Statistics NCfH, vol. 10: Vital Health Stat; 2013

45. CDC. Adult participation in aerobic and muscle-strengthening physical activities-United States. Centers for Disease Control and Prevention, MMWR Morb Mortal Wkly Rep 2013. 2011;62(17):326-30.

46. Bennie JA, Chau JY, van der Ploeg HP, Stamatakis E, Do A, Bauman A. The prevalence and correlates of sitting in European adults - a comparison of 32 Eurobarometer-participating countries. Int J Behav Nutr Phys Act. 2013;10(1):107.

47. Bauman A, Ainsworth BE, Sallis JF, Hagstromer M, Craig CL, Bull FC, et al. The descriptive epidemiology of sitting. A 20-country comparison using the International Physical Activity Questionnaire (IPAQ). Am J Prev Med. 2011; 41(2):228-35.

48. 4363.0.55.001 - Australian Health Survey: Users' Guide, 2011-13 [http://www. abs.gov.au/ausstats/abs@.nsf/Latestproducts/4363.0.55.

001 Main\%20Features12011-

13?opendocument\&tabname=Summary\&prodno $=4363.0 .55$.

001\&issue $=2011-13 \&$ num $=\& v i e w=]$.

49. ABS. National Nutrition and Physical Activity Survey Questionnaire. In., vol. http://www.ausstats.abs.gov.au/ausstats/subscriber.nsf/0/ 734DF823586D5AD9CA257B8E0014A387/\$File/ national\%20nutrition\%20and\%20physical\%20activity\%20survey\%20201112\%20questionnaire.pdf. Canberra: Australian Bureau of Statistics; 2012.

50. Heesch KC, Hill RL, van Uffelen JG, Brown WJ. Are Active Australia physical activity questions valid for older adults? J Sci Med Sport. 2011;14(3):233-7.

51. Brown W, Burton N, Marshall A, Miller Y. Reliability and validity of a modified self-administered version of the Active Australia physical 2 activity survey in a sample of mid-age women. Aust N Z J Public Health. 2008;32(6):535-41.

52. AlHW. In: Welfare AloHa, editor. The Active Australia Survey: a Guide and Manual for Implementation, Analysis and Reporting. Canberra: ACT; 2003.

53. Yore MM, Ham SA, Ainsworth BE, Kruger J, Reis JP, Kohl 3rd H, et al. Reliability and validity of the instrument used in BRFSS to assess physical activity. Med Sci Sports Exerc. 2007;39(8):1267-74.

54. Schoenborn CA, Adams PF, JA P. Health behaviors of adults: United States, 2008-2010, Data from the National Health Survey. In: Vital and Health Statistics. Washington: DC Center for Health Statistics; 2013.

55. Healy GN, Clark BK, Winkler EA, Gardiner PA, Brown WJ, Matthews CE. Measurement of adults' sedentary time in population-based studies. Am J Prev Med. 2011;41(2):216-27.

56. Marshall AL, Miller YD, Burton NW, Brown WJ. Measuring total and domainspecific sitting: a study of reliability and validity. Med Sci Sports Exerc. 2010; 42(6):1094-102.

57. Chau JY, Grunseit A, Midthjell K, Holmen J, Holmen TL, Bauman AE, Van der Ploeg HP. Sedentary behaviour and risk of mortality from all-causes and cardiometabolic diseases in adults: evidence from the HUNT3 population cohort. Br J Sports Med 2013

58. Brown WJ, Bauman AE, Bull FC, Burton NW. Development of evidencebased physical activity recommendations for adults (18-64 years): report prepared for the Australian Government Department of Health, August 2012. 2013.

59. Dunstan D, Healy G, Owen N, Salmon J, Ridgers N, Okley T, et al. Action area 5: Prolonged sitting. In: Blueprint for an active Australia. Melbourne: National Heart Foundation of Australia; 2014.

60. Bauman A, Ford I, Armstrong T. Trends in population levels of reported physical activity in Australia, 1997, 1999 and 2000. Canberra 2001.

61. Australian Institute of Health and Welfare. In: Welfare AloHa, editor. Cardiovascular disease, diabetes and chronic kidney disease Australian facts: Risk factors. 4th ed. Canberra: ACT; 2015.

62. Nakamura Y, Harada K. Promotion of Strength Training. In: Physical Activity, Exercise, Sedentary Behavior and Health. 2015.

63. Grøntved A, Pan A, Mekary RA, Stampfer M, Willett WC, Manson JE, et al. Muscle-strengthening and conditioning activities and risk of type 2 diabetes: a prospective study in two cohorts of US women. PLoS Med. 2014;11(1):e1001587. 
64. Flack KD, Davy KP, Hulver MW, Winett RA, Frisard MI, Davy BM. Aging, resistance training, and diabetes prevention. Journal of aging research 2001. 2011.

65. Orr R, Raymond J, Fiatarone Singh M. Efficacy of progressive resistance training on balance performance in older adults: a systematic review of randomized controlled trials. Sports Med. 2008;38(4):317-43.

66. Nakamura Y, Harada K. Promtion of Strength Training. In: Kanosue K, Oshima S, Cao ZB, Oka K, editors. Physical Activity, Exercise, Sedentary Behavior and Health. Tokyo: Springer Verlag, Japan; 2015. p. 29-42.

67. Sallis JF, Hovell MF. Determinants of exercise behavior. Exerc Sport Sci Rev. 1990;18:307-30.

68. Trost S, Owen N, Bauman A, Sallis J, Brown W. Correlates of adult's participation in physical activity: review and update. Med Sci Sports Exerc 2002;34(12):1996-2001.

69. Inoue M, Iso H, Yamamoto S, Kurahashi N, Iwasaki M, Sasazuki S, et al. Daily total physical activity level and premature death in men and women: results from a large-scale population-based cohort study in Japan (JPHC study). Ann Epidemiol. 2008;18(7):522-30.

70. Rhodes RE, Mark RS, Temmel CP. Adult sedentary behavior: a systematic review. Am J Prev Med. 2012;42(3):e3-28.

71. Chastin SF, Buck C, Freiberger E, Murphy M, Brug J, Cardon G, et al. Systematic literature review of determinants of sedentary behaviour in older adults: a DEDIPAC study. Int J Behav Nutr Phys Act. 2015;12:127.

72. Sallis J, Owen N. Physical Activity and Behavioral Medicine. Thousand Oaks: Sage Publications; 1999.

73. Pedišić Ž, Bauman A. Accelerometer-based measures in physical activity surveillance: current practices and issues. British journal of sports medicine 2014:bjsports-2013-093407.

74. Healy GN, Dunstan DW, Salmon J, Cerin E, Shaw JE, Zimmet PZ, et al. Breaks in sedentary time - beneficial associations with metabolic risk. Diabetes Care. 2008;31(4):661-6.

75. Pedisic Z, Grunseit A, Ding D, Chau JY, Banks E, Stamatakis E, et al. High sitting time or obesity: Which came first? Bidirectional association in a longitudinal study of 31,787 Australian adults. Obesity (Silver Spring). 2014; 22(10):2126-30.

76. van Uffelen JG, Watson MJ, Dobson AJ, Brown WJ. Sitting time is associated with weight, but not with weight gain in mid-aged Australian women. Obesity (Silver Spring). 2010;18(9):1788-94.

\section{Submit your next manuscript to BioMed Central and we will help you at every step:}

- We accept pre-submission inquiries

- Our selector tool helps you to find the most relevant journal

- We provide round the clock customer support

- Convenient online submission

- Thorough peer review

- Inclusion in PubMed and all major indexing services

- Maximum visibility for your research

Submit your manuscript at www.biomedcentral.com/submit

C) Biomed Central 